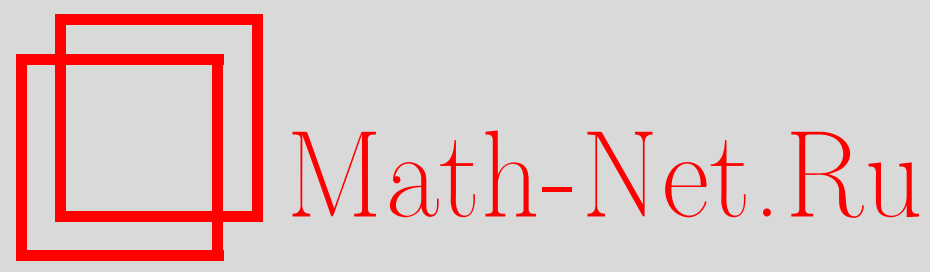

Ф. М. Аблаев, А. В. Васильев, О реализации булевых функций в квантовых ветвящихся программах методом отпечатков, Дискрет. матем., 2009, том 21, выпуск 4, 3-19

DOI: https://doi.org/10.4213/dm1067

Использование Общероссийского математического портала Math-Net.Ru подразумевает, что вы прочитали и согласны с пользовательским соглашением http://www . mathnet.ru/rus/agreement

Параметры загрузки:

IP : 35.174 .16 .151

26 апреля 2023 г., $12: 14: 13$ 


\title{
О реализации булевых функций в квантовых ветвящихся программах методом отпечатков
}

\author{
() 2009 г. Ф. М. Аблаев, А. В. Васильев
}

В работе представлен разработанный нами метод отпечатков вычисления булевых функций в квантовых моделях вычислений. Этот метод является развитием техники fingerprinting.

Применение метода отпечатков демонстрируется на примере вычисления функции $\mathrm{MOD}_{m}$ в классе квантовых OBDD (забывающих один раз читающих ветвящихся программ). Далее возможности метода отпечатков демонстрируются на примере реализации функции "равенство" в квантовой модели коммуникационных вычислений с рефери (SMP communication model) и на примере распознавания языков в квантовых автоматах.

Все приведенные реализации булевых функций в различных квантовых моделях вычислений (OBDD, коммуникационная модель SMP и конечный автомат) являются асимптотически оптимальными.

Работа выполнена при поддержке Российского фонда фундаментальных исследований, проект 08-07-00449, а также Университета Турку.

Авторы благодарят Юхани Кархумяки за приглашение в Университет Турку и плодотворные обсуждения предмета статьи.

\section{1. Введение}

Задача физической реализации полномасштабного квантового вычислителя - открытая проблема для современной технологии. Дальнейшее уменьшение масштабов при производстве электронных схем приведет к тому, что функционирование их компонент будет происходить по законам квантовой механики. Поэтому по мере развития квантовых нанотехнологий в классические компьютеры будут включаться квантовые компоненты и отдельные модули. В настоящее время в физических центрах проводятся интенсивные исследования по расчетам и разработке транзисторов, учитывающих законы квантовой механики. При преодолении современных технологических трудностей построения квантовых вычислительных систем квантовые технологии откроют возможности реализации массивного распараллеливания вычислений, известного как квантовый параллелизм. Один из первых эффективных квантовых алгоритмов (полиномиальный квантовый алгоритм факторизации числа [1]) продемонстрировал потенциальные преимущества квантовых моделей вычислений для ряда задач по сравнению с классическими.

Помимо физических проблем в области исследований и разработок квантовых моделей вычислений требуют своего разрешения серьезные математические вопросы. Важной открытой математической проблемой является описание класса задач, решаемых на квантовых моделях вычислений более эффективно, чем на классических. 
Важным вопросом остается вопрос определения адекватных сложностных характеристик для сопоставления квантовых и классических моделей вычислений. В данной работе за такую меру сложности мы берем число кубит и бит, необходимых для реализации квантовой и классической вычислительной модели соответственно. В данной статье мы предлагаем схемное представление квантовых ветвящихся программ (КВП), для которых эта мера сложности является наиболее адекватной.

Графовый подход при определении классических ветвящихся программ использовался во множестве публикаций (см., например, книгу [2]) и является исторически наиболее распространенным. Квантовые ветвящиеся программы впервые были определены именно в терминах графов [3].

Алгебраический подход для определения классических ветвящихся программ использовался, по-видимому, впервые в работе [4]. Для определения квантовых ветвящихся программ алгебраический подход является естественным [5, 6, 7].

В данной работе мы приводим алгебраические определения ветвящихся программ и предлагаем определение квантовой ветвящейся программы (КВП) в виде квантовой схемы с возможностью использования классических бит в качестве управляющих для унитарных преобразований.

Для данной модели мы разрабатываем метод отпечатков (quantum fingerprinting technique). Подобные техники позволяют представлять исходный объект при помощи компактного образа (отпечатка), устраивать экономные по памяти вычисления и достаточно достоверно извлекать правильный результат. Как правило, такой подход используется в вероятностных и квантовых алгоритмах для проверки равенства таких объектов как двоичные наборы, полиномы, матрицы и т. д. при помощи сравнения их отпечатков. Впервые такая техника построения эффективных вероятностных алгоритмов была предложена Фрейвалдом [8], см. также книгу [9].

Для квантовой коммуникационной модели техника отпечатков была впервые явно сформулирована в [10]. Эта статья положила начало серии подобного рода результатов в области квантовых коммуникаций. Неявно метод отпечатков также применялся для квантовых конечных автоматов в [11] (и позже в [12]) и квантовых ветвящихся программ в $[6,7]$.

В данной работе мы обобщаем подход из [12] и явно определяем технику отпечатков для квантовых вычислительных устройств. Наши построения используют на каждом шаге однотипные преобразования и могут быть представлены наглядной квантовой схемой.

В модели квантовых один раз читающих ветвящихся программ (квантовых OBDD, QOBDD) рассматривается булева функция $\mathrm{MOD}_{m}$, проверяющая, кратно ли число единиц во входном наборе параметру $m$. Строится алгоритм, вычисляющий эту функцию с экспоненциальной экономией по квантовой памяти (числу кубит). Благодаря нижней оценке на размер QOBDD из [6] можно утверждать, что наш алгоритм асимптотически оптимален по числу кубит.

Данная статья устроена следующим образом. В следующем разделе приводятся необходимые сведения о квантовых ветвящихся программах и предлагается их схемное представление. Раздел 3 содержит описание предложенной техники в обобщенном виде, а также доказательство существования набора необходимых параметров. Далее наш метод применяется для построения оптимальной квантовой OBDD для булевой функции $\mathrm{MOD}_{m}$.

Доказанное в разделе 3 утверждение позволяет также обобщить построения квантового автомата, распознающего делимость (регулярный язык $L_{m}$ ) из [12]. Об этом упоминается в разделе 4 .

В том же разделе разработанный подход применяется для решения проблемы равенства в некоторой трехсторонней коммуникационной модели (simultaneous message passing 
model without shared keys). Полученный протокол дает по существу ту же сложность и вероятность ошибки, что и [10].

Результаты работы докладывались на XV международной конференции «Проблемы теоретической кибернетики» [13].

\section{2. Предварительные сведения}

\section{1. Основы квантовых вычислений}

Теория квантовых вычислений опирается на постулаты квантовой механики. Приведем необходимые сведения из книги [15].

Пространство состояний. Согласно первому постулату, с любой изолированной физической системой можно связать гильбертово пространство, называемое пространством состояний системы. Система полностью описывается единичным вектором из пространства состояний, называемым вектором состояния (или, сокращенно, состоянием).

Квантовый бит (кубит) является ключевым понятием теории квантовых вычислений. Он рассматривается как квантово-механическая система, описываемая комплекснозначными векторами двумерного гильбертова пространства $\mathscr{H}^{2}$, то есть

$$
|\psi\rangle=\alpha|0\rangle+\beta|1\rangle
$$

где векторы $|0\rangle$ и $|1\rangle$ образуют ортонормированный базис в $\mathscr{H}^{2}$, а комплексные числа $\alpha$ и $\beta$ удовлетворяют условию $|\alpha|^{2}+|\beta|^{2}=1$. Таким образом, в отличие от классического бита кубит может одновременно находиться в суперпозиции своих базисных состояний $|0\rangle$ и $|1\rangle$ с амплитудами $\alpha$ и $\beta$ соответственно.

Обозначим через $|i\rangle$ вектор из $\mathscr{H}^{d}$, у которого 1 стоит на позиции $i$, а все остальные компоненты нулевые. Система векторов $|1\rangle,|2\rangle, \ldots,|i\rangle, \ldots,|d\rangle$ образует ортонормированный базис в пространстве $\mathscr{H}^{d}$. Будем также обозначать указанные векторы через $|00 \ldots 0\rangle,|00 \ldots 1\rangle, \ldots,|\operatorname{bin}(i-1)\rangle, \ldots,|11 \ldots 1\rangle$, где $\operatorname{bin}(i)$ - это двоичное представление числа $i$.

Линейные комбинации $\sum_{i} \alpha_{i}|i\rangle$ принято называть суперпозициями базисных состояний $|i\rangle$ с амплитудами $\alpha_{i}$.

Составные системы. При рассмотрении квантового регистра (то есть совокупности отдельных кубитов), квантовая механика постулирует, что пространство состояний такой составной системы будет описываться тензорным произведением пространств состояний подсистем. Например, если $n$ кубитов находятся в состояниях $\left|\psi_{1}\right\rangle,\left|\psi_{2}\right\rangle, \ldots,\left|\psi_{n}\right\rangle$, то состояние $|\psi\rangle$ квантового регистра, состоящего из этих кубитов, будет выражаться через тензорное произведение их состояний:

$$
|\psi\rangle=\left|\psi_{1}\right\rangle \otimes\left|\psi_{2}\right\rangle \otimes \ldots \otimes\left|\psi_{n}\right\rangle .
$$

Такие состояния называются разложимыми.

В то же время существуют так называемые неразложимые или запутанные состояния квантовых регистров, которые не могут быть представлены тензорным произведением состояний отдельных кубитов. Такими состояниями являются, например, ЭПР-пары или состояния Белла, играющие ключевую роль в квантовой телепортации и сверхплотном кодировании. 
В общем случае регистр из $n$ квантовых бит описывается суперпозицией

$$
|\psi\rangle=\sum_{|i\rangle \in \mathscr{H}^{2^{n}}} \alpha_{i}|i\rangle .
$$

Причем условие нормировки требует, чтобы

$$
\|\psi\|_{2}^{2}=\sum_{i}\left|\alpha_{i}\right|^{2}=1
$$

Динамика изменения. Следующий постулат гласит, что динамика изменения состояния замкнутой квантовой системы описывается унитарными преобразованиями. Другими словами, состояние системы $\left|\psi_{1}\right\rangle$ в момент времени $t_{1}$ связано с состоянием $\left|\psi_{2}\right\rangle$ в момент времени $t_{2}$ следующим образом: $\left|\psi_{1}\right\rangle=U\left|\psi_{2}\right\rangle$, где $U-$ унитарный оператор, зависящий только от $t_{1}$ и $t_{2}$.

Измерение. Хотя кубиты и могут одновременно находиться в различных состояниях, информация об этом доступна лишь опосредованно - путем измерения состояния. Причем это вероятностный процесс, то есть, измеряя суперпозицию $\sum_{i} \alpha_{i}|i\rangle$, мы получим любое из состояний $|i\rangle$ с вероятностью $\left|\alpha_{i}\right|^{2}$. Такое извлечение информации называется измерением в стандартном вычислительном базисе. В данной работе рассматриваются только такие измерения.

В разделе 4.1 нам потребуется вариант квантового измерения, называемый проективным измерением. Для задания такого измерения достаточно перечислить полный набор ортогональных проекторов $P_{m}$, которые удовлетворяют отношениям $\sum_{m} P_{m}=I$ и $P_{m} P_{m^{\prime}}=\delta_{m m^{\prime}} P_{m}$. Вероятность исхода $m$ в таком случае можно выразить как

$$
\mathbf{P}(m)=\| P_{m}|\psi\rangle \|_{2}^{2},
$$

а состоянием системы после измерения будет $P_{m}|\psi\rangle / \sqrt{\mathbf{P}(m)}$.

Сфера Блоха. Сфера Блоха - это геометрическое представление состояния кубита в виде точки на единичной трехмерной сфере, она является обобщением представления комплексного числа $z$, для которого $|z|^{2}=1$, в виде точки на единичной окружности. Действие многих однокубитных операторов удобно иллюстрируется на сфере Блоха.

Рассмотрим кубит в состоянии $|\psi\rangle=\alpha|0\rangle+\beta|1\rangle$. Так как $|\alpha|^{2}+|\beta|^{2}=1$, амплитуды можно записать в виде $\alpha=e^{i \gamma} \cos \theta^{\prime}, \beta=e^{i \varphi^{\prime}} \sin \theta^{\prime}$, где $0 \leqslant \gamma \leqslant 2 \pi, 0 \leqslant \theta^{\prime} \leqslant \pi / 2$ и $0 \leqslant \varphi^{\prime} \leqslant \pi-$ вещественные числа, и пусть $\varphi=\varphi^{\prime}-\gamma$. Далее, это состояние можно переписать в виде

$$
|\psi\rangle=e^{i \gamma}\left(\cos \theta^{\prime}|0\rangle+e^{i \varphi} \sin \theta^{\prime}|1\rangle\right) .
$$

Множитель $e^{i \gamma}$ можно игнорировать, так как $\left|e^{i \gamma}\right|^{2}=1$, и поэтому он никак не влияет на результат измерения состояния кубита. Следовательно, состояние можно переписать в виде

$$
|\psi\rangle=\cos \theta^{\prime}|0\rangle+e^{i \varphi} \sin \theta^{\prime}|1\rangle .
$$

Пусть $e^{i \varphi} \sin \theta^{\prime}=x+i y$, обозначим $z=\cos \theta^{\prime}$. Тогда $x^{2}+y^{2}+z^{2}=1$, то есть каждое состояние кубита соответствует точке на единичной сфере, задаваемой полярными координатами $\theta^{\prime}$ и $\varphi$. 
Заметим, что противоположная точка на сфере с координатами $\pi-\theta^{\prime}$ и $\pi+\varphi$ соответствует состоянию

$$
\begin{aligned}
\left|\psi^{\prime}\right\rangle & =\cos \left(\pi-\theta^{\prime}\right)|0\rangle+e^{i(\pi+\varphi)} \sin \left(\pi-\theta^{\prime}\right)|1\rangle \\
& =-\cos \theta^{\prime}|0\rangle-e^{i \varphi} \sin \theta^{\prime}|1\rangle=-|\psi\rangle .
\end{aligned}
$$

Поэтому достаточно рассматривать только верхнюю полусферу $\left(0 \leqslant \theta^{\prime} \leqslant \pi / 2\right)$, так как состояния, соответствующие точкам нижней полусферы, отличаются только на множитель $e^{i \pi}=-1$, не имеющий наблюдаемого эффекта.

Вводя обозначение $\theta=2 \theta^{\prime}$, получаем, что каждому состоянию

$$
|\psi\rangle=\cos \frac{\theta}{2}|0\rangle+e^{i \varphi} \sin \frac{\theta}{2}|1\rangle,
$$

где $0 \leqslant \gamma \leqslant 2 \pi, 0 \leqslant \theta \leqslant \pi$, соответствует точка на трехмерной единичной сфере, называемой сферой Блоха.

Унитарные операторы. Множество унитарных операторов континуально, но их можно сколь угодно точно представить в виде произведения унитарных операций из некоторого конечного универсального набора [15].

Таковым набором является, например, совокупность следующих операторов:

$$
\begin{gathered}
H=\frac{1}{\sqrt{2}}\left(\begin{array}{cc}
1 & 1 \\
1 & -1
\end{array}\right), \quad S=\left(\begin{array}{ll}
1 & 0 \\
0 & i
\end{array}\right), \quad T=\frac{\pi}{8}=e^{i \pi / 8}\left(\begin{array}{ccc}
e^{-i \pi / 8} & 0 \\
0 & e^{i \pi / 8}
\end{array}\right), \\
\mathrm{CNOT}=\left(\begin{array}{llll}
1 & 0 & 0 & 0 \\
0 & 1 & 0 & 0 \\
0 & 0 & 0 & 1 \\
0 & 0 & 1 & 0
\end{array}\right) .
\end{gathered}
$$

Известно, что любое однокубитное унитарное преобразование можно с произвольной точностью $\varepsilon$ представить в виде произведения $O\left(\log ^{c}(1 / \varepsilon)\right)$ операторов $H$ и $\pi / 8$ (константа $c$ приблизительно равна 2), а произвольное унитарное преобразование, действующее на $q$ кубитах, может быть представлено в виде произведения $O\left(q^{2} 4^{q}\right)$ однокубитных и CNOT операторов. Фазовый оператор $S$ включается в стандартный набор для реализации контролируемых операторов и для организации помехоустойчивых вычислений.

Следующие унитарные операторы называются операторами вращения, потому что соответствуют вращениям на угол $\theta$ вокруг осей $\hat{x}, \hat{y}$ и $\hat{z}$ сферы Блоха:

$$
\begin{aligned}
& R_{\hat{x}}(\theta)=\left(\begin{array}{cc}
\cos \frac{\theta}{2} & -i \sin \frac{\theta}{2} \\
-i \sin \frac{\theta}{2} & \cos \frac{\theta}{2}
\end{array}\right) ; \\
& R_{\hat{y}}(\theta)=\left(\begin{array}{cc}
\cos \frac{\theta}{2} & -\sin \frac{\theta}{2} \\
\sin \frac{\theta}{2} & \cos \frac{\theta}{2}
\end{array}\right) \\
& R_{\hat{z}}(\theta)=\left(\begin{array}{cc}
e^{-i \theta / 2} & 0 \\
0 & e^{i \theta / 2}
\end{array}\right)
\end{aligned}
$$


Контролируемые операторы, реализующие некоторое преобразование при выполнении определенного условия, являются основой для квантового параллелизма. Пусть кубиты $\left|c_{1}\right\rangle,\left|c_{2}\right\rangle, \ldots,\left|c_{q}\right\rangle$ находятся в некотором базисном состоянии. Оператор $C^{q}(U)$, управляемый кубитами $\left|c_{1}\right\rangle,\left|c_{2}\right\rangle, \ldots,\left|c_{q}\right\rangle$, можно задать уравнением вида

$$
C^{q}(U)\left|c_{1}\right\rangle\left|c_{2}\right\rangle \ldots\left|c_{q}\right\rangle|\psi\rangle=\left|c_{1}\right\rangle\left|c_{2}\right\rangle \ldots\left|c_{q}\right\rangle U^{c_{1} c_{2} \ldots c_{q}}|\psi\rangle
$$

то есть унитарное преобразование $U$ применяется к целевому кубиту $|\psi\rangle$, если управляющие кубиты $\left|c_{1}\right\rangle\left|c_{2}\right\rangle \ldots\left|c_{q}\right\rangle$ находятся в базисном состоянии $|1\rangle^{\otimes q}$, в противном случае применяется тождественное преобразование $I$. В квантовых схемах из функциональных элементов такие операторы принято обозначать следующим образом:

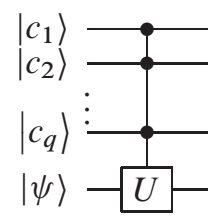

Известно, что произвольный контролируемый оператор $C^{q}(U)$, где $U-$ однокубитное унитарное преобразование, можно реализовать при помощи $O\left(q^{2}\right)$ однокубитных и CNOT операторов.

Контролируемые операторы можно обобщить на случай, когда для срабатывания управляющие кубиты должны находиться в состоянии $|0\rangle$, а не $|1\rangle$. Для этого до и после применения контролируемого оператора соответствующий кубит инвертируется при помощи оператора отрицания $X$. Кроме того, вводится специальное обозначение

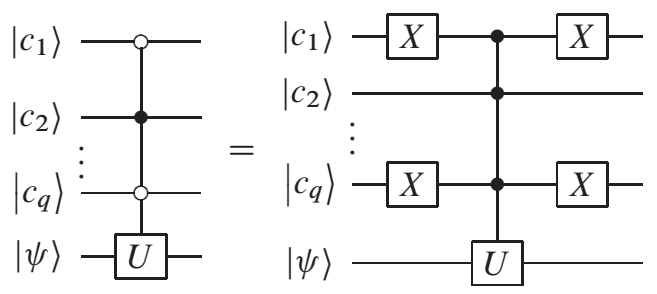

Пусть $|1\rangle,|2\rangle, \ldots,|i\rangle, \ldots,|d\rangle$ - ортонормированный базис в $d$-мерном гильбертовом пространстве, где $d=2^{q}$.

Обозначим через $C_{i}^{q}(U)$ (или $C_{i}(U)$ ) контролируемый оператор с $q$ управляющими и одним целевым кубитом, причем преобразование $U$ применяется к целевому кубиту, если управляющие кубиты находились в состоянии $|i\rangle$. На схемах будем обозначать такой оператор следующим образом:

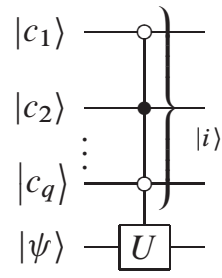

В данной работе мы будем использовать контролируемые вращения вокруг оси $|y\rangle$ сферы Блоха и будем обозначать их $C_{i}(R)$. 


\section{2. Определение квантовых ветвящихся программ}

Определение линейной ветвящейся программы является обобщением понятия квантовой ветвящейся программы, приведенного в [6]. Детерминированные и квантовые забывающие ветвящиеся программы являются частными случаями линейных ветвящихся программ. Пусть $\mathbf{V}^{d}-d$-мерное векторное пространство. Обозначим через $|\psi\rangle$ и $\langle\psi|$ векторы-столбцы и векторы-строки из $\mathbf{V}^{d}$, а также будем использовать обозначения $\left\langle\psi_{1} \mid \psi_{2}\right\rangle$ для скалярного произведения. Будем писать $\psi$, когда нам не важно, столбец это или строка.

Обозначим через $|i\rangle$ вектор из $\mathbf{V}^{d}$, у которого на $i$-й позиции 1 , а все остальные компоненты нулевые. Очевидно, что система векторов $|1\rangle, \ldots,|d\rangle$ образует ортонормированный базис в пространстве $\mathbf{V}^{d}$.

Определение 1. Линейная ветвящаяся программа $P$ ширины $d$ и длины $l((d, l)$-ЛВП) над векторным пространством $\mathbf{V}^{d}$ есть тройка $P=\left(T,\left|\psi_{0}\right\rangle\right.$, Ассерt), где $T$ есть последовательность из $l$ инструкций: $T_{j}=\left(x_{i_{j}}, U_{j}(0), U_{j}(1)\right)$ определяется переменной $x_{i_{j}}$, считываемой на шаге $j$, и $d \times d$ матрицами $U_{j}(0)$ и $U_{j}(1)$.

Векторы $|\psi\rangle \in \mathbf{V}^{d}$ называются состояниями (векторами состояний) программы $P$, $\left|\psi_{0}\right\rangle \in \mathbf{V}^{d}$ есть начальное состояние $P$, а Accept $\subseteq\{|1\rangle, \ldots,|d\rangle\}$ есть принимающее множество.

Вычисления на входном наборе $\sigma=\left(\sigma_{1}, \ldots, \sigma_{n}\right) \in\{0,1\}^{n}$ происходят следующим образом:

(1) $P$ начинает вычисления в исходном состоянии $\left|\psi_{0}\right\rangle$;

(2) $j$-я инструкция $P$ считывает переменную $x_{i_{j}}$ и применяет преобразование $U_{j}=U_{j}\left(\sigma_{i_{j}}\right)$ к текущему состоянию $|\psi\rangle$, переводящее программу $P$ в состояние $\left|\psi^{\prime}\right\rangle=U_{j}\left(x_{i_{j}}\right)|\psi\rangle$;

(3) конечным состоянием программы является

$$
|\psi(\sigma)\rangle=\left(\prod_{j=l}^{1} U_{j}\left(\sigma_{i_{j}}\right)\right)\left|\psi_{0}\right\rangle .
$$

Ширину линейной ветвящейся программы $P$ будем обозначать width $(P)$.

Детерминированные ветвящиеся программы. Детерминированная ветвящаяся программа есть линейная ветвящаяся программа над векторным пространством $\{0,1\}^{d}$. Состояние $|\psi\rangle$ такой программы есть булев вектор, содержащий ровно одну 1. Матрицы $U_{j}$ соответствуют подстановкам степени $d$ и поэтому содержат ровно одну 1 в каждом столбце. Для ветвящихся программ над конечными группами то же выполняется и для строк, то есть $U_{j}$ являются перестановочными матрицами.

Квантовые ветвящиеся программы. Мы определяем квантовую ветвящуюся программу (КВП) как линейную ветвящуюся программу над гильбертовым пространством $\mathscr{H}^{d}$. Для такой программы $|\psi\rangle$ - комплекснозначные векторы с $\||\psi\rangle \|_{2}=1$, а $U_{j}$ являются комплекснозначными унитарными матрицами.

После $l$-го (последнего) шага вычислений измеряется состояние $P$

$$
\left|\psi_{\sigma}\right\rangle=U_{l}\left(\sigma_{i_{l}}\right) U_{l-1}\left(\sigma_{i_{l-1}}\right) \ldots U_{1}\left(\sigma_{i_{1}}\right)\left|\psi_{0}\right\rangle .
$$


Измерение задается диагональной проекционной матрицей $M$, где $M_{i i}=1$, если $|i\rangle \in$ Accept, и $M_{i i}=0$ в противном случае. Вероятность $\mathbf{P}_{\text {Aсcерt }}(\sigma)$ принятия входного набора $\sigma$ задается равенством

$$
\mathbf{P}_{\text {Accept }}(\sigma)=\| M\left|\psi_{\sigma}\right\rangle \|_{2}^{2}
$$

Говорят, что КВП $P$ вычисляет булеву функцию $f$ с изолированной точкой сечения, если существует $\varepsilon \in(0,1 / 2]$ такое, что для любого $\sigma \in f^{-1}(1)$ вероятность принятия этого набора программой $P$ не меньше $1 / 2+\varepsilon$, а для любого $\sigma \in f^{-1}(0)$ вероятность принятия не превышает $1 / 2-\varepsilon$.

В частности, говорят, что КВП $P$ вычисляет булеву функцию $f$ с односторонней ошибкой, если существует $\varepsilon>0$ такое, что для любого $\sigma \in f^{-1}(1)$ вероятность принятия этого набора программой $P$ равна 1 , а для любого $\sigma \in f^{-1}(0)$ вероятность принятия не превышает $1-\varepsilon$.

Заметим, что это модель с одним измерением (measure-once), аналогичная модели конечных квантовых автоматов в работе [14], в которой состояние системы изменяется унитарным образом за исключением финального измерения состояния. Можно было бы также разрешить измерения в процессе вычисления, представляя состояние матрицей плотности $\rho$ и полагая $U_{j}$ супероператорами, но мы не рассматриваем это в данной работе.

\section{Один раз читающие ветвящиеся программы.}

Определение 2. ЛВП $P$ называется OBDD или один раз читающей ЛВП, если каждая переменная $x \in\left\{x_{1}, \ldots, x_{n}\right\}$ появляется в последовательности инструкций $T$ программы $P$ не более одного раза.

ЛВП являются забывающими по построению, и поэтому данное определение согласуется с формулировкой понятия OBDD в терминах графов. Обозначим через QOBDD квантовые один раз читающие ветвящиеся программы, а для детерминированных будем использовать OBDD.

Справедливость следующей нижней оценки для ширины QOBDD была доказана в [6].

Теорема 1. Пусть $\varepsilon \in(0,1 / 2)$ и пусть $f\left(x_{1}, \ldots, x_{n}\right)-$ булева функиия, вычисляемая с ограниченной ошибкой квантовой один раз читающей ветвящейся программой $Q$. Тогда

$$
\operatorname{width}(Q)=\Omega(\log \text { width }(P))
$$

где Р есть минимальная по ширине OBDD, вычисляющая $f\left(x_{1}, \ldots, x_{n}\right)$.

Схемное представление. КВП может рассматриваться как квантовая схема, дополненная возможностью считывать классические биты в качестве контролирующих для унитарных операторов. Таким образом, любая квантовая схема есть КВП, которая существенно 
не зависит от своих классических входов.

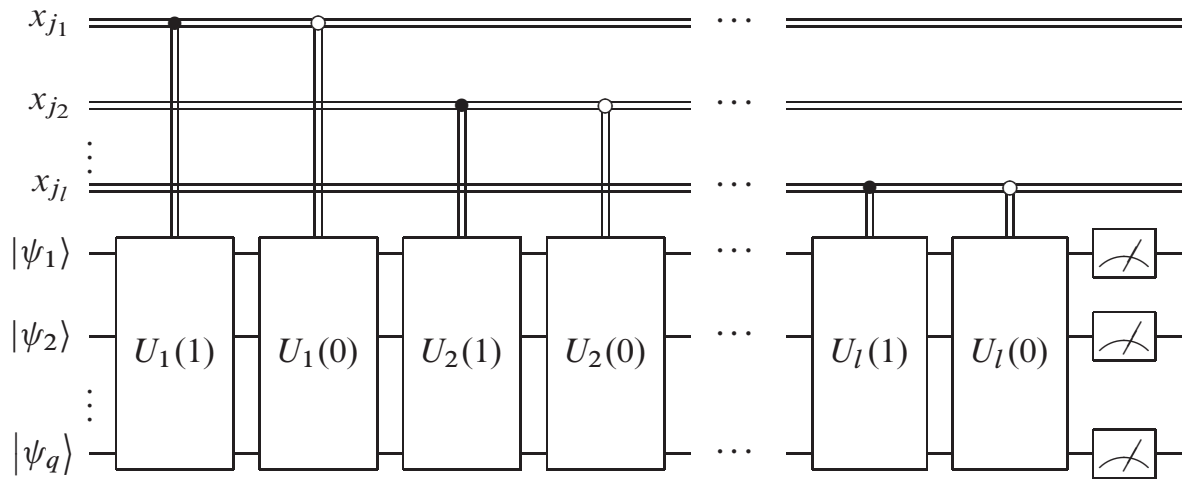

Здесь $x_{j_{1}}, \ldots, x_{j_{l}}$ - последовательность переменных (необязательно различных), обозначающих классические управляющие сигналы. Согласно принятой в литературе по квантовым схемам нотации классическое управление обозначается на схеме двойными проводами, а квантовое - одиночными.

Заметим, что для квантовой ветвящейся программы в схемном представлении явным образом проявляется еще одна мера сложности - число кубит $q$, необходимое для физической реализации соответствующей квантовой системы с классическим управлением. Согласно постулатам квантовой механики для реализации квантовой ветвящейся программы ширины $d$ (системы с $d$ состояниями) потребуется как минимум $\log d$ кубит.

Определение 3. Назовем квантовую ветвящуюся программу $q$-кубитной, если она может быть реализована в виде классически управляемой квантовой системы, основанной на $q$ кубитах.

\section{3. Метод отпечатков}

Метод отпечатков (fingerprinting) - это техника, которая позволяет представлять объекты (слова в некотором конечном алфавите) их образами (отпечатками, fingerprints), значительно более компактными, чем оригиналы. Кроме того, они позволяют достаточно достоверно (с односторонней ошибкой) извлекать информацию о входном наборе. Техника отпечатков, описанная в работе [10], дает возможность строить оптимальный коммуникационный протокол в некоторой трехсторонней модели SMP для определения тождества двух двоичных наборов. В данной работе мы представляем технику отпечатков, адаптированную для квантовых ветвящихся программ. Она основывается на работе [12], тем самым обобщая построения указанной статьи. Разработанный метод применяется для построения оптимальной КВП, вычисляющей булеву функцию $\mathrm{MOD}_{m}$. В заключение демонстрируется, что этот подход для проблемы равенства в модели SMP приводит к результату, аналогичному результату из [10].

Наш подход обладает следующими свойствами.

- Он ориентирован на модели с классическим управлением, а значит, и на КВП.

- Образы входных слов легко построить, используются только контролируемые вращения вокруг оси $\hat{y}$ сферы Блоха на различные углы и преобразования Адамара (понятие сферы Блоха и унитарные преобразования подробно описаны в книге [15]). 
- Доказанная лемма гарантирует существование хорошего множества параметров, что позволяет ограничить вероятность ошибки сверху некоторой константой $0<\varepsilon<1$.

Техника отпечатков. Для решаемой задачи выбираются целое $m \geqslant 2$ и допустимая вероятность ошибки $\varepsilon>0$. Затем фиксируется

$$
t=\lceil(2 / \varepsilon) \ln 2 m\rceil
$$

и строится отображение $g:\{0,1\}^{n} \rightarrow \mathbf{Z}$, описывающее некоторое свойство входного набора. Для произвольного двоичного набора $\sigma=\sigma_{1} \ldots \sigma_{n}$ порождается ее отпечаток $\left|h_{\sigma}\right\rangle$, объединяющий в себе $t$ однокубитных отпечатков $\left|h_{\sigma}^{i}\right\rangle$ :

$$
\begin{aligned}
\left|h_{\sigma}^{i}\right\rangle & =\cos \frac{2 \pi k_{i} g(\sigma)}{m}|0\rangle+\sin \frac{2 \pi k_{i} g(\sigma)}{m}|1\rangle, \\
\left|h_{\sigma}\right\rangle & =\frac{1}{\sqrt{t}} \sum_{i=1}^{t}|i\rangle\left|h_{\sigma}^{i}\right\rangle,
\end{aligned}
$$

то есть последний кубит в квантовом регистре поворачивается на $t$ различных углов (вокруг оси $\hat{y}$ сферы Блоха), определяемых параметрами $k_{i} \in\{1, \ldots, m-1\}$.

Предлагаемая техника нацелена на достоверное распознавание равенства нулю значения $g(\sigma)$. Для этой цели параметры $k_{i} \in\{1, \ldots, m-1\}$ для всех $i=1, \ldots, t$ выбираются специальным образом, исходя из следующего определения.

Определение 4. Множество $K=\left\{k_{1}, \ldots, k_{t}\right\}$ называется хорошим для целого числа $b \neq 0 \bmod m$, если

$$
\frac{1}{t^{2}}\left(\sum_{i=1}^{t} \cos \frac{2 \pi k_{i} b}{m}\right)^{2}<\varepsilon
$$

Левая часть неравенства соответствует квадрату амплитуды базисного состояния $|0\rangle^{\otimes \log t}|0\rangle$ после применения оператора $H^{\otimes \log t} \otimes I$ к отпечатку $\left|h_{\sigma}\right\rangle$. Применительно к нашим алгоритмам такое множество гарантирует, что вероятность ошибки будет ограничена константой, меньшей 1.

Следующая лемма утверждает существование хорошего множества и развивает идеи соответствующего утверждения из [12].

Лемма 1. Сушествует множество $K$, где $|K|=t=\lceil(2 / \varepsilon) \ln 2 m\rceil$, которое является хорошим для всех иельхх $b \neq 0 \bmod m$.

Доказательство. Используя неравенство Азумы (смотри, например, [9]), докажем, что случайно выбранное множество $K$ будет хорошим с положительной вероятностью.

Пусть $1 \leqslant b \leqslant m-1$, и пусть $K$ будет множеством $t$ независимых случайных величин, равномерно распределенных на $\{0, \ldots, m-1\}$.

Определим случайные величины

$$
X_{i}=\cos \frac{2 \pi k_{i} b}{m}, \quad Y_{k}=\sum_{i=1}^{k} X_{i}
$$

Докажем, что неравенство Азумы применимо к последовательности $Y_{0}=0, Y_{1}, Y_{2}, Y_{3}, \ldots$, то есть последняя является мартингалом с ограниченными разностями. 
Во-первых, необходимо доказать, что $\mathbf{E} Y_{k}<\infty$. Из определения $X_{i}$ следует, что

$$
\mathbf{E} X_{i}=\frac{1}{m} \sum_{j=0}^{m-1} \cos \frac{2 \pi j b}{m} .
$$

Взвешенная сумма $m$-х корней из единицы

$$
\frac{1}{m} \sum_{j=0}^{m-1} \exp \left(\frac{2 \pi j b}{m} i\right)=\frac{1}{m} \frac{\exp (2 \pi i b m / m)-1}{\exp (2 \pi i b / m)-1}=0,
$$

так как $b$ не кратно $m$.

Математическое ожидание $\mathbf{E} X_{i}$ есть как раз действительная часть предыдущей суммы и поэтому равняется 0. Следовательно,

$$
\mathbf{E} Y_{k}=\sum_{i=1}^{k} \mathbf{E} X_{i}=0
$$

Во-вторых, нужно показать, что условное ожидаемое значение следующего испытания при известных предыдущих исходах равняется последнему исходу. Нетрудно видеть, что

$$
\begin{aligned}
\mathbf{E}\left[Y_{k+1} \mid Y_{1}, \ldots, Y_{k}\right] & =\frac{1}{m} \sum_{j=0}^{m-1}\left(Y_{k}+\cos \frac{2 \pi j b}{m}\right) \\
& =Y_{k}+\frac{1}{m} \sum_{j=0}^{m-1} \cos \frac{2 \pi j b}{m}=Y_{k} .
\end{aligned}
$$

Так как

$$
\left|Y_{k+1}-Y_{k}\right|=\left|X_{k+1}\right| \leqslant 1, \quad k \geqslant 0,
$$

применение неравенства Азумы приводит к неравенству

$$
\mathbf{P}\left(\left|Y_{t}-Y_{0}\right| \geqslant \lambda\right)=\mathbf{P}\left(\left|\sum_{i=1}^{t} X_{i}\right| \geqslant \lambda\right) \leqslant 2 \exp \left(-\frac{\lambda^{2}}{2 t}\right) .
$$

Поэтому вероятность того, что $K$ не будет хорошим для $g \in\{1 \ldots m-1\}$ не превосходит

$$
\mathbf{P}\left(\left|\sum_{i=1}^{t} X_{i}\right| \geqslant \sqrt{\varepsilon} t\right) \leqslant 2 \exp \left(-\frac{\varepsilon t}{2}\right) \leqslant \frac{1}{m}
$$

для $t=\lceil(2 / \varepsilon) \ln 2 m\rceil$.

Таким образом, вероятность того, что построенное множество не будет хорошим хотя бы для одного $1 \leqslant b \leqslant m-1$ не больше $(m-1) / m<1$. Следовательно, существует множество, которое будет хорошим для всех $1 \leqslant b \leqslant m-1$. Это множество также будет хорошим для всех $b \neq 0 \bmod m$, так как

$$
\cos \frac{2 \pi k(b+j m)}{m}=\cos \frac{2 \pi k b}{m} .
$$

Мы используем этот результат для разрабатываемой техники, выбирая множество $K=\left\{k_{1}, \ldots, k_{t}\right\}$ хорошим для всех $b=g(\sigma) \neq 0$. Это позволяет достоверно отличить те входные наборы, которые $g$ отображает в 0 по модулю $m$, от остальных. 


\section{1. Вычисление функции $\mathrm{MOD}_{m}$ методом отпечатков}

Рассмотрим булеву функцию $\mathrm{MOD}_{m}$ : на входном наборе $\sigma=\sigma_{1} \ldots \sigma_{n} \in\{0,1\}^{n}$ она равна единице тогда и только тогда, когда число единиц в наборе $\sigma$ кратно $m$, где $m \geqslant 2$ - целое число.

Теорема 2. Для любого $\varepsilon \in(0,1)$ существует $O(\log \log m)$-кубитная QOBDD $P$ (один раз читающая $O(\log \log m)$-кубитная КВП), вычисляющзая функцию $\mathrm{MOD}_{m}$ с односторонней оиибкой $\varepsilon$.

Доказательство. Выберем $\varepsilon>0$ и зафиксируем $t=\lceil(2 / \varepsilon) \ln 2 m\rceil$. Если $t$ не является степенью 2, то можно положить

$$
t=2^{\lceil\log (\lceil(2 / \varepsilon) \ln 2 m\rceil)\rceil} \geqslant\lceil(2 / \varepsilon) \ln 2 m\rceil .
$$

Согласно приведенному доказательству леммы 1 , вероятность существования хорошего множества при этом не уменьшится.

Ниже приведена $\log 2 t$-кубитная QOBDD $Q$ для функции $\mathrm{MOD}_{m}$ в виде схемы.

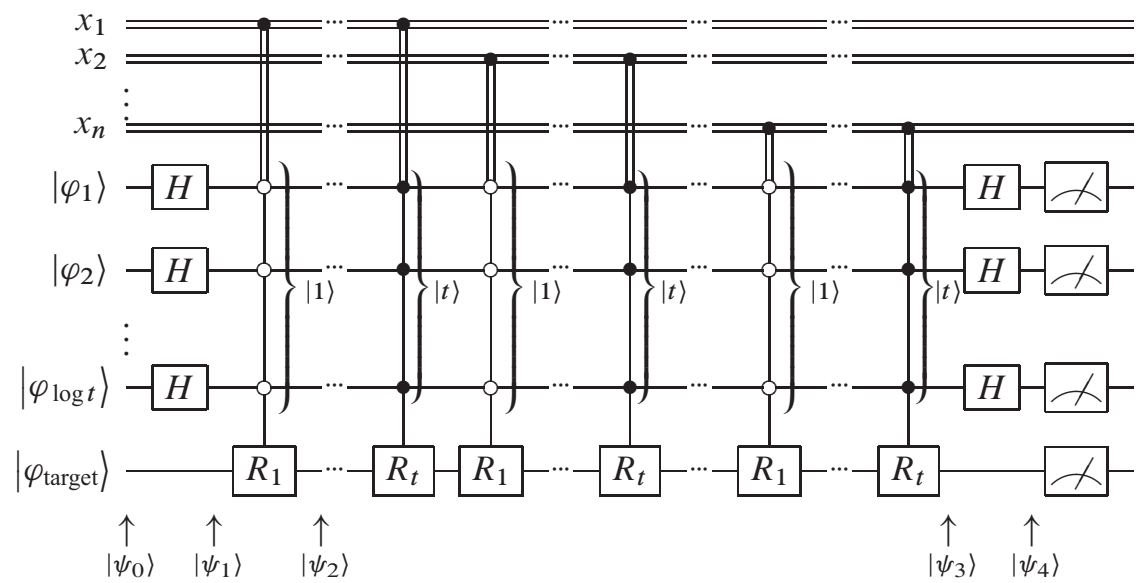

Докажем, что КВП $Q$ вычисляет $\mathrm{MOD}_{m}$ с односторонней ошибкой $\varepsilon$. Рассмотрим работу алгоритма на произвольном входном наборе $\sigma=\sigma_{1} \ldots \sigma_{n} \in\{0,1\}^{n}$.

Изначально

$$
\left|\psi_{0}\right\rangle=\left|\varphi_{1}\right\rangle\left|\varphi_{2}\right\rangle \ldots\left|\varphi_{\log t}\right\rangle\left|\varphi_{\text {target }}\right\rangle=|0\rangle^{\otimes \log t}|0\rangle
$$

Унитарные преобразования

$$
R_{i}=R_{\hat{y}}\left(\frac{4 \pi k_{i}}{m}\right)
$$

для всех $i \in\{1, \ldots, t\}$ являются вращениями целевого кубита $\left|\varphi_{\text {target }}\right\rangle$ на угол $4 \pi k_{i} / m$ вокруг оси $\hat{y}$ сферы Блоха, а множество параметров $K=\left\{k_{1}, \ldots, k_{t}\right\}$ выбрано хорошим согласно определению 4.

На первом этапе преобразования Адамара переводят квантовый регистр в состояние

$$
\begin{aligned}
\left|\psi_{1}\right\rangle & =\left(H^{\otimes \log t} \otimes I\right)|0\rangle^{\otimes \log t}|0\rangle=H|0\rangle \ldots H|0\rangle|0\rangle \\
& =\left(\frac{|0\rangle+|1\rangle}{\sqrt{2}}\right) \ldots\left(\frac{|0\rangle+|1\rangle}{\sqrt{2}}\right)|0\rangle=\frac{1}{\sqrt{t}} \sum_{i=1}^{t}|i\rangle|0\rangle,
\end{aligned}
$$


то есть переводят состояние первых $\log t$ кубитов в равноамплитудную комбинацию всех базисных состояний.

Далее, если считывается 0, то состояние не меняется, то есть применяется тождественное преобразование $I$. Когда же на вход поступает единица, применяются контролируемые операторы $C_{i}\left(R_{i}\right)$ : каждый из них нетривиальным образом действует только на состояния вида $|i\rangle|\varphi\rangle$, переводя их в $|i\rangle R_{i}|\varphi\rangle$. Следовательно, если $\sigma_{1}=1$, то система перейдет из состояния $\left|\psi_{1}\right\rangle$ в

$$
\left|\psi_{2}\right\rangle=\frac{1}{\sqrt{t}} \sum_{i=1}^{t}|i\rangle R_{i}|0\rangle=\frac{1}{\sqrt{t}} \sum_{i=1}^{t}|i\rangle\left(\cos \frac{2 \pi k_{i}}{m}|0\rangle+\sin \frac{2 \pi k_{i}}{m}|1\rangle\right) .
$$

Таким образом, для входного набора $\sigma \in\{0,1\}^{n}$, интерпретируемого как унарное представление числа $g(\sigma)=\sum_{i=1}^{n} \sigma_{i}$, программа $Q$ создает (шаг за шагом в процессе считывания набора $\sigma$ ) его отпечаток:

$$
\left|\psi_{3}\right\rangle=\frac{1}{\sqrt{t}} \sum_{i=1}^{t}|i\rangle\left(\cos \frac{2 \pi k_{i} g(\sigma)}{m}|0\rangle+\sin \frac{2 \pi k_{i} g(\sigma)}{m}|1\rangle\right) .
$$

Затем оператор $H^{\otimes \log t} \otimes I$ переводит $\left|\psi_{3}\right\rangle$ в состояние

$$
\begin{aligned}
\left|\psi_{4}\right\rangle & =\left(H^{\otimes \log t} \otimes I\right) \frac{1}{\sqrt{t}} \sum_{i=1}^{t}|i\rangle\left(\cos \frac{2 \pi k_{i} g(\sigma)}{m}|0\rangle+\sin \frac{2 \pi k_{i} g(\sigma)}{m}|1\rangle\right) \\
& =\frac{1}{\sqrt{t}} \sum_{i=1}^{t} H^{\otimes \log t}|i\rangle\left(\cos \frac{2 \pi k_{i} g(\sigma)}{m}|0\rangle+\sin \frac{2 \pi k_{i} g(\sigma)}{m}|1\rangle\right) .
\end{aligned}
$$

Пусть $i_{1} i_{2} \ldots i_{\log t}-$ двоичное разложение числа $i-1$. Тогда

$$
|i\rangle=\left|i_{1} i_{2} \ldots i_{\log t}\right\rangle
$$

и

$$
\begin{aligned}
H^{\otimes \log t}|i\rangle & =H\left|i_{1}\right\rangle H\left|i_{2}\right\rangle \ldots H\left|i_{\log t}\right\rangle \\
& =\left(\frac{|0\rangle+(-1)^{i_{1}}|1\rangle}{\sqrt{2}}\right)\left(\frac{|0\rangle+(-1)^{i_{2}}|1\rangle}{\sqrt{2}}\right) \ldots\left(\frac{|0\rangle+(-1)^{i_{\log t}}|1\rangle}{\sqrt{2}}\right) \\
& =\frac{1}{\sqrt{t}}\left(|0\rangle^{\otimes \log t}+\sum_{j=2}^{t} \xi_{i j}|j\rangle\right),
\end{aligned}
$$

где $\xi_{i j}= \pm 1$.

Далее в $\left|\psi_{4}\right\rangle$ нас будет интересовать только амплитуда при нулевом базисном состоянии, поэтому, подставляя выражение для $H^{\otimes \log t}|i\rangle$, можем записать это состояние в виде

$$
\left|\psi_{4}\right\rangle=\left(\frac{1}{t} \sum_{i=1}^{t} \cos \left(2 \pi k_{i} g(\sigma) / m\right)|0\rangle^{\otimes \log t}|0\rangle+\gamma|0\rangle^{\otimes \log t}|1\rangle+\sum_{i=2}^{t}|i\rangle\left(\alpha_{i}|0\rangle+\beta_{i}|1\rangle\right)\right.
$$

для некоторых амплитуд $\gamma, \alpha_{i}$ и $\beta_{i}$. 
Итоговое состояние $\left|\psi_{4}\right\rangle$ измеряется в стандартном вычислительном базисе. Набор $\sigma$ принимается программой, если результатом измерения будет состояние $|0\rangle^{\otimes \log t}|0\rangle$, в противном случае $\sigma$ отвергается. Из построений видно, что для произвольного набора $\sigma$ вероятность принятия равна

$$
\mathbf{P}_{\text {Accept }}(\sigma)=\frac{1}{t^{2}}\left(\sum_{i=1}^{t} \cos \left(2 \pi k_{i} g(\sigma) / m\right)^{2}\right.
$$

Таким образом, если $\operatorname{MOD}_{m}(\sigma)=1$, то есть $g(\sigma)=0 \bmod m$, то $\mathbf{P}_{\text {Accept }}(\sigma)=1$. A если $\operatorname{MOD}_{m}(\sigma)=0(g(\sigma) \neq 0 \bmod m)$, то вероятность наблюдения состояния $|0\rangle^{\otimes \log t}|0\rangle$ меньше $\varepsilon$ благодаря выбору множества параметров $k_{1}, \ldots, k_{t}$.

В данном алгоритме применялась техника, описанная в разделе 3 , где полагалось, что

$$
g(x)=\sum_{i=1}^{n} x_{i}
$$

а параметр $m$ равен модулю в определении $\mathrm{MOD}_{m}$. Заметим, что число кубит, используемых данной конструкцией, равно $q=\log t+1=O(\log \log m)$, а ширина программы есть $2^{q}=O(\log m)$. Этот результат асимптотически оптимален в силу теоремы 1 и того факта, что любая детерминированная OBDD для $\mathrm{MOD}_{m}$ требует ширины $\Omega(m)$.

\section{4. Метод отпечатков для коммуникационных вычислений и автоматов}

В этом разделе мы демонстрируем реализацию квантовых вычислений методом отпечатков в коммуникационной модели с рефери SMP (simultaneous message passing model) и в квантовых конечных автоматах.

\section{1. Распознавание равенства в коммуникационной модели SMP}

Рассмотрим проблему равенства в трехсторонней коммуникационной модели SMP без общего ключа, предложенной в [16]. В этой модели двое участников, обладающих неограниченными вычислительными возможностями, получают двоичные наборы $\sigma$ и $\gamma$ соответственно, но не могут взаимодействовать друг с другом. Они лишь могут отправить сообщение арбитру, который должен определить значение вычисляемой протоколом функции, опираясь лишь на полученные сообщения. Коллективная задача участников вычислить значение $f(\sigma, \gamma)$ при минимально возможной длине сообщений. Проблема равенства состоит в проверке совпадения двоичных наборов $\sigma$ и $\gamma$.

В [10] было показано, что при наличии у участников общего ключа достаточно сообщений константной длины. Однако при отсутствии такого ключа длина сообщений будет $\Theta(\log n)$, где $n-$ длина входных наборов. Заметим, что в вероятностном случае сложность рассматриваемой проблемы есть $\Theta(\sqrt{n})$, то есть в данном случае использование квантовых методов дает экспоненциальный выигрыш в коммуникационной сложности.

Следующий результат улучшает оценку вероятности ошибки протокола по сравнению с результатом, полученном в [10].

Теорема 3. Для любого $\varepsilon \in(0,1)$ существует квантовый протокол, который решает проблему равенства в модели SMP с односторонней ошибкой $(1+\varepsilon) / 2$, используя $O(\log n)$ кубит для сообщений. 
Доказательство. Применим технику из раздела 3 в совокупности со своп-тестом из [10]. Положим параметр $m$ равным $2^{n}$, где $|\sigma|=|\gamma|=n$, зададим отображение

$$
g(x)=\sum_{i=1}^{n} 2^{i-1} x_{i}
$$

как десятичное значение входного двоичного числа, зафиксируем $0<\varepsilon<1$ и выберем хорошее множество из $t=\lceil(2 / \varepsilon) \ln 2 m\rceil$ параметров $K=\left\{k_{1}, \ldots, k_{t}\right\}$.

Участники создают для своих двоичных наборов квантовые регистры в состоянии $\left|h_{\sigma}\right\rangle$ и $\left|h_{\gamma}\right\rangle$ соответственно, где

$$
\begin{aligned}
& \left|h_{\sigma}\right\rangle=\frac{1}{\sqrt{t}} \sum_{i=1}^{t}|i\rangle\left(\cos \frac{2 \pi k_{i} g(\sigma)}{m}|0\rangle+\sin \frac{2 \pi k_{i} g(\sigma)}{m}|1\rangle\right) \\
& \left|h_{\gamma}\right\rangle=\frac{1}{\sqrt{t}} \sum_{i=1}^{t}|i\rangle\left(\cos \frac{2 \pi k_{i} g(\gamma)}{m}|0\rangle+\sin \frac{2 \pi k_{i} g(\gamma)}{m}|1\rangle\right) .
\end{aligned}
$$

Затем они пересылают их арбитру, который работает по следующей схеме:

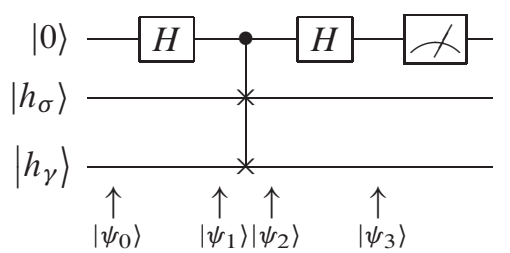

Преобразование Адамара переводит квантовый регистр, находящийся в состояние $\left|\psi_{0}\right\rangle=|0\rangle\left|h_{\sigma}\right\rangle\left|h_{\gamma}\right\rangle$, в состояние

$$
\left|\psi_{1}\right\rangle=\left(\frac{|0\rangle+|1\rangle}{\left|h_{\sigma}\right\rangle} \sqrt{2}\right)\left|h_{\sigma}\right\rangle\left|h_{\gamma}\right\rangle .
$$

Далее применяется преобразование C(SWAP), обменивающее состояния целевых кубитов, если управляющий кубит находится в состоянии $|1\rangle$. Следовательно,

$$
\left|\psi_{2}\right\rangle=\frac{1}{\sqrt{2}}|0\rangle\left|h_{\sigma}\right\rangle\left|h_{\gamma}\right\rangle+\frac{1}{\sqrt{2}}|1\rangle\left|h_{\gamma}\right\rangle \mid h_{\sigma} .
$$

Второе преобразование Адамара переводит квантовый регистр в состояние

$$
\begin{aligned}
\left|\psi_{3}\right\rangle & =\frac{1}{\sqrt{2}}\left(\frac{|0\rangle+|1\rangle}{\sqrt{2}}\right)\left|h_{\sigma}\right\rangle\left|h_{\gamma}\right\rangle+\frac{1}{\sqrt{2}}\left(\frac{|0\rangle-|1\rangle}{\sqrt{2}}\right)\left|h_{\gamma}\right\rangle\left|h_{\sigma}\right\rangle \\
& =\frac{1}{2}|0\rangle\left(\left|h_{\sigma}\right\rangle\left|h_{\gamma}\right\rangle+\left|h_{\gamma}\right\rangle\left|h_{\sigma}\right\rangle\right)+\frac{1}{2}|1\rangle\left(\left|h_{\sigma}\right\rangle\left|h_{\gamma}\right\rangle-\left|h_{\gamma}\right\rangle\left|h_{\sigma}\right\rangle\right) .
\end{aligned}
$$

Измеряя первый кубит в стандартном вычислительном базисе (используя ортогональные проекторы $P_{0}=|0\rangle\langle 0|$ и $\left.P_{1}=|1\rangle\langle 1|\right)$, арбитр принимает входной набор, если получено состояние $|0\rangle$. Согласно постулатам квантовой механики вероятность этого события равна скалярному квадрату проекции $P_{0} \otimes I \otimes I\left|\psi_{3}\right\rangle$, то есть

$$
\begin{aligned}
\mathbf{P}_{\text {Accept }}(\sigma, \gamma) & =\left(P_{0} \otimes I \otimes I\left|\psi_{3}\right\rangle, P_{0} \otimes I \otimes I\left|\psi_{3}\right\rangle\right) \\
& =\left(\frac{1}{2}|0\rangle\left(\left|h_{\sigma}\right\rangle\left|h_{\gamma}\right\rangle+\left|h_{\gamma}\right\rangle\left|h_{\sigma}\right\rangle\right), \frac{1}{2}|0\rangle\left(\left|h_{\sigma}\right\rangle\left|h_{\gamma}\right\rangle+\left|h_{\gamma}\right\rangle\left|h_{\sigma}\right\rangle\right)\right) .
\end{aligned}
$$


Скалярное произведение тензорного произведения векторов есть

$$
\left.\left(\left|\psi_{1}\right\rangle \otimes\left|\psi_{2}\right\rangle,\left|\varphi_{1}\right\rangle \otimes\left|\varphi_{2}\right\rangle\right)=\left(\left|\psi_{1},\right| \varphi_{1}\right\rangle\right)\left(\left|\psi_{2}\right\rangle,\left|\varphi_{2}\right\rangle\right)=\left\langle\psi_{1} \mid \varphi_{1}\right\rangle\left\langle\psi_{2} \mid \varphi_{2}\right\rangle,
$$

и для любого состояния $|\psi\rangle$ выполняется равенство $\langle\psi \mid \psi\rangle=1$, поэтому

$$
\begin{aligned}
\mathbf{P}_{\text {Accept }}(\sigma, \gamma) & =\frac{1}{4}\langle 0 \mid 0\rangle\left(\left\langle h_{\sigma} \mid h_{\sigma}\right\rangle\left\langle h_{\gamma} \mid h_{\gamma}\right\rangle+2\left\langle h_{\sigma} \mid h_{\gamma}\right\rangle\left\langle h_{\gamma} \mid h_{\sigma}\right\rangle++\left\langle h_{\gamma} \mid h_{\gamma}\right\rangle\left\langle h_{\sigma} \mid h_{\sigma}\right\rangle\right) \\
& =\frac{1}{4}\left(2+2\left|\left\langle h_{\sigma} \mid h_{\gamma}\right\rangle\right|^{2}\right)=\frac{1}{2}\left(1+\left|\left\langle h_{\sigma} \mid h_{\gamma}\right\rangle\right|^{2}\right) .
\end{aligned}
$$

Далее, подставляя выражения для $\left|h_{\sigma}\right\rangle$ и $\left|h_{\gamma}\right\rangle$, получаем, что

$$
\begin{aligned}
\mathbf{P}_{\text {Accept }}(\sigma, \gamma) & =\frac{1}{2}+\frac{1}{2 t^{2}}\left|\sum_{i=1}^{t} \cos \frac{2 \pi k_{i} g(\sigma)}{m} \cos \frac{2 \pi k_{i} g(\gamma)}{m}+\sin \frac{2 \pi k_{i} g(\sigma)}{m} \sin \frac{2 \pi k_{i} g(\gamma)}{m}\right|^{2} \\
& =\frac{1}{2}+\frac{1}{2 t^{2}}\left|\sum_{i=1}^{t} \cos \frac{2 \pi k_{i}(g(\sigma)-g(\gamma))}{m}\right|^{2} .
\end{aligned}
$$

Другими словами, когда $\sigma=\gamma$, вероятность принятия равна 1 , а вероятность получить состояние $|0\rangle$, когда $\sigma \neq \gamma$, ограничена так, что

$$
\mathbf{P}_{\text {Accept }}(\sigma, \gamma)<\frac{1}{2}+\frac{1}{2} \varepsilon<1
$$

Число кубит, передаваемых арбитру, равно

$$
2 \log 2 t=O(\log \log m)=O(\log n) .
$$

В [10] также была доказана нижняя оценка $\Omega(\log n)$ для рассматриваемой проблемы, поэтому наш подход является асимптотически оптимальным, как и предложенный в [10].

\section{2. Распознавание делимости в конечных квантовых автоматах}

Используя доказательство из раздела 3, можно утверждать, что результат статьи [12] о распознавании регулярного языка $L_{m}$ односторонним конечным квантовым автоматом может быть распространен на случай произвольных целых $m \geqslant 2$.

Указанный язык определяется как

$$
L_{m}=\left\{a^{i} \mid i \text { кратно } m\right\}
$$

для некоторого $m \geqslant 2$. В [12] приведена конструкция квантового автомата, распознающего данный язык для простых $m$.

Теорема 4. Для любого $\varepsilon>0$ сущеествует квантовый конечный автомат с $O(\log m)$ состояниями, распознающий язык $L_{m}$ с вероятностью $1-\varepsilon$.

Доказательство. Рассмотрим квантовый конечный автомат с множеством состояний $Q=\left\{q_{1,0}, q_{1,1}, \ldots, q_{t, 0}, q_{t, 1}\right\}$, где $\left\{q_{1,0}\right\}$ - принимающее состояние, а все остальные отвергающие. Пусть его начальным состоянием является $\frac{1}{\sqrt{t}} \sum_{i=1}^{t}\left|q_{i, 0}\right\rangle$. Входной алфавит состоит из единственного символа $a$, а входное слово начинается после маркера \# и заканчивается маркером \&. Функция переходов на входном символе $a$ переводит состояние 
$\left|q_{i, 0}\right\rangle$ в состояние $\left(\cos \left(\left(2 \pi k_{i} / p\right)\left|q_{i, 0}\right\rangle\right)+\sin \left(2 \pi k_{i} / p\right) \mid q_{i, 1}\right)$, а состояние $\left|q_{i, 1}\right\rangle$ в состояние $\left(-\sin \left(\left(2 \pi k_{i} / p\right)\left|q_{i, 0}\right\rangle+\cos \left(\left(2 \pi k_{i} / p\right)\left|q_{i, 1}\right\rangle\right)\right.\right.$. При считывании правого маркера \$ реализуется преобразование $H^{\otimes t} \otimes I$, то есть состояния $\left|q_{i, j}\right\rangle$ переводятся в $(1 / \sqrt{t})\left|q_{1, j}\right\rangle$ плюс некоторая комбинация остальных состояний. Заметим, что при считывании слова $a^{l}$ автомат перейдет в состояние $\left.\left(\frac{1}{\sqrt{t}} \sum_{i=1}^{t} \cos \frac{2 \pi k_{i} l}{m}\right)\left|q_{1,0}\right\rangle+\gamma q_{1,1}\right\rangle+\sum_{i=2}^{t}\left(\alpha_{i}\left|q_{i, 0}\right\rangle+\beta_{i}\left|q_{i, 1}\right\rangle\right)$, где $\gamma, \alpha_{i}$ и $\beta_{i}$ - некоторые амплитуды, значения которых для нас не важны. Таким образом, если $l=0 \bmod m$, то вероятность получить состояние $\left|q_{1,0}\right\rangle$ равна 1 ; в противном случае выбор хорошего множества $K=\left\{k_{1}, \ldots, k_{t}\right\}$, где $t=\lceil(2 / \varepsilon) \ln 2 m\rceil=O(\log m)$, гарантирует, что вероятность ошибочного принятия входного набора не превзойдет $\varepsilon$.

\section{Список литературы}

1. Shor P. W., Polynomial-time algorithms for prime factorization and discrete logarithms on a quantum computer. SIAM J. Comput. (1997) 26, 1484-1509.

2. Wegener I., Branching programs and binary decision diagrams. SIAM Press, Philadelphia, 2000.

3. Nakanishi M., Hamaguchi K., Kashiwabara T., Ordered quantum branching programs are more powerful than ordered probabilistic branching programs under a bounded-width restriction. Lect. Notes Comput. Sci. (2000) 1858, 467-476.

4. Barrington D. A., Bounded-width polynomial-size branching programs recognize exactly those languages in $N C^{1}$. J. Comput. Syst. Sci. (1989) 38, 150-164.

5. Sauerhoff M., Sieling D., Quantum branching programs and space-bounded nonuniform quantum complexity. Theor. Comput. Sci. (2005) 334, 177-225.

6. Ablayev F., Gainutdinova A., Karpinski M., On computational power of quantum branching programs. Lect. Notes Comput. Sci. (2001) 2138, 59-70.

7. Ablayev F., Gainutdinova A., Karpinski M., Moore C., Pollette C., On the computational power of probabilistic and quantum branching programs of constant width. Inf. Comput. (2005) 203, 145-162.

8. Freivalds R., Fast probabilistic algorithms. Lect. Notes Comput. Sci. (1979) 74, 57-69.

9. Motwani R., Raghavan P., Randomized algorithms. Cambridge University Press, Cambridge, 1995.

10. Buhrman H., Cleve R., Watrous J., Wolf R., Quantum fingerprinting. Phys. Rev. Lett. (2001) 87 (16), 167902.

11. Ambainis A., Freivalds R., 1-way quantum finite automata: strengths, weaknesses and generalization. In: Proc. 39th FOCS. Washington, DC, 1998, pp. 332-342.

12. Ambainis A., Nahimovs N., Improved constructions of quantum automata. Lect. Notes Comput. Sci. (2008) 5106, 47-56.

13. Аблаев Ф. М., Васильев А. В., О вычислениях в квантовых ветвящихся программах методом характерных признаков. В сб.: Тез. докл. XV Международной конф. «Проблемы теоретической кибернетики», Казань, 2008, с. 1 (in Russian).

14. Moore C., Crutchfield J. P., Quantum automata and quantum grammars. Theor. Comput. Sci. (2000) 237, 275-306.

15. Nielsen M. A., Chuang I. L., Quantum computation and quantum information. Cambridge University Press, Cambridge, 2000.

16. Yao A., Some complexity questions related to distributive computing. In: Proc. 11th STOC, 1979, pp. 209-213. 